\title{
A Two-Step Approach to Hallucinating Faces: Global Parametric Model and Local Nonparametric Model
}

\author{
Ce Liu* $^{* \dagger}$ Heung-Yeung Shum ${ }^{\dagger}$ Chang-Shui Zhang* \\ ${ }^{*}$ State Key Lab of Intelligent Technology and Systems, Dept. of Automation, Tsinghua University, Beijing 100084, China \\ ${ }^{\dagger}$ Visual Computing Group, Microsoft Research China, Sigma Building, Zhi-Chun Road, Beijing 100080, China
}

\begin{abstract}
In this paper, we study face hallucination or synthesizing a high-resolution face image from a low-resolution input, with the help of a large collection of other highresolution face images. We develop a two-step statistical modeling approach that integrates both a global parametric model and a local nonparametric model. First, we derive a global linear model to learn the relationship between the high-resolution face images and their smoothed and down-sampled lower resolution ones. Second, the residual between an original high-resolution image and the reconstructed high-resolution image by learned linear model is modeled by a patch-based nonparametric Markov network, to capture the high-frequency content of faces. By integrating both global and local models, we can generate photorealistic face images. Our approach is demonstrated by extensive experiments with high-quality hallucinated faces.
\end{abstract}

\section{Introduction}

Super-resolution techniques in computer vision infer the missing high-resolution image from the low-resolution input. Low-resolution is equivalent to low-frequency and high-resolution consists of high, middle and low frequency bands. There are in general two classes of super-resolution techniques: reconstruction-based (from input images alone) and learning-based (from other images). Of particular interest is face hallucination, or learning high-resolution face images from low-resolution ones. Face hallucination is a term coined by Baker and Kanade [1], which implies the high-frequency part of face image must be purely fabricated. Hallucinating faces is particularly challenging because people are so familiar with faces.

We argue that a successful face hallucination algorithm should meet the following three constraints:

1) Sanity constraint. The result must be very close to the input image when smoothed and down-sampled.

2) Global constraint. The result must have common characteristics of a human face, e.g. eyes, mouth and nose, symmetry, etc.

3) Local constraint. The result must have specific characteristics of this face image with photorealistic local features.

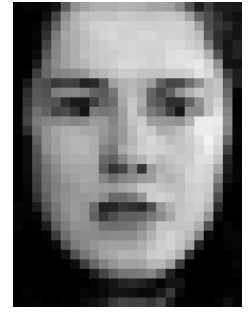

(a) Input $24 \times 32$

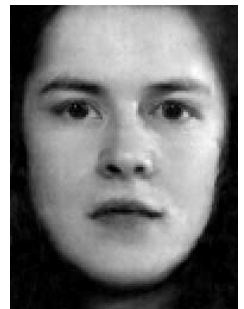

(b) Hallucinated result

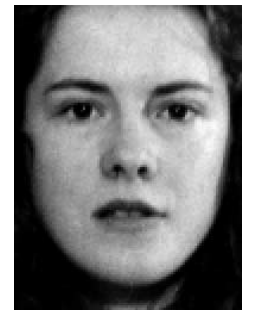

(c) Original $96 \times 128$
Figure 1. An example of face hallucination using our approach. The hallucinated image (b) is locally different from, but globally similar to the original high-resolution one (c).

The first requirement can be easily made. For example, it can be simply formulated as a linear constraint on the result. The second and third ones are apparently much harder. It is important to satisfy the three requirements altogether to hallucinate faces with good quality. Without the constraint on specific face features, the result would be too smooth, close to the average face. On the other hand, without a global face similarity constraint, the result could be noisy.

Such global and local constraints lead us to a hybrid approach. We combine a global parametric model which generalizes well with common faces, with a local nonparametric model which learns local textures from example faces. This approach can also be applied to modeling other visual patterns, in particular for the structural objects with both global coherence such as illumination, contour and symmetry, and precise local textures like skin and hair.

We incorporate all the constraints in a statistical face model and find the maximum a posteriori (MAP) solution for the hallucinated face. An example of a hallucinated image from an input low-resolution image with our approach is shown in Figure 1. The sanity constraint is simply modeled as a Gaussian distribution or a soft constraint. The global constraint assumes a Gaussian distribution learnt by principal component analysis (PCA). The local constraint utilizes a patch-based nonparametric Markov network to learn the statistical relationship between the global face image and the local features. A two-step approach is then used in hallucinating faces. First, an optimal global face image is obtained in the eigen-space when constraints 1) and 2) are satisfied. Second, an optimal local feature image is inferred 
from the optimal global image by minimizing the energy of the Markov network with constraint 3) applied. The sum of the global and local images forms the final result.

After reviewing related work in Section 2, we introduce the details of our model in Section 3. Many convincing examples are shown in Section 4. Section 5 gives discussion and conclusion.

\section{Related Work}

Most learning-based super-resolution algorithms such as $[3,6,7]$ assume homogeneous (stationary) Markov random fields (MRFs) for images. Let $L$ denote an image lattice, and $\mathbf{v}$ a certain position on the lattice with $I_{\mathbf{v}}$ as the pixel value. $I_{\mathrm{v}}^{-}$represents all pixels on $L$ other than $I_{\mathbf{v}}$. $L$ is a Markov random field if

$$
p\left(I_{\mathbf{v}} \mid I_{\mathbf{v}}^{-}\right)=p\left(I_{\mathbf{v}} \mid N_{\mathbf{v}}\right),
$$

where $N_{\mathbf{v}}$ is the neighborhood of $\mathbf{v}$ [14]. This definition indicates that a pixel only relies on the pixels in its neighborhood. Further, $L$ is a homogeneous MRF if for any positions $\mathbf{v}$ and $\mathbf{u} \in L$, their conditional density functions are identical, i.e.,

$$
p\left(I_{\mathbf{v}} \mid N_{\mathbf{v}}\right)=p\left(I_{\mathbf{u}} \mid N_{\mathbf{u}}\right),
$$

which also implies that their neighborhoods have equal size.

Proposed for texture synthesis, the multi-resolution nonparametric sampling method developed by De Bonet [3] indeed infers the high-frequency features from the lowfrequency features named the parent structure. It is demonstrated that in a homogeneous MRF, the high-frequency component locally depends on the low-frequency part. Freeman and Pasztor [6] proposed a parametric Markov network to learn the statistics between the "scene" and "image", as a framework for handling low-level vision tasks. It can be applied in super resolution work if the scene and image are high- and low-frequency bands respectively. Recently Hertzmann et al. [7] generalizes local feature transform methods in "Image Analogies". Given a pair of training images, an analogous image is inferred from the input by the local similarity between the training pair. It can also fulfill super resolution objectives if the training pairs are high- and low-resolution images, respectively.

All of above methods do local feature transfer/inference with low-level vision. They perform well in hallucinating texture-like images, but would fail in hallucinating structural visual patterns such as human faces. To broaden the application to face hallucination, the homogeneous MRF assumption (2) has to be abandoned, leading to the work by Baker and Kanade [1]. They only follow the MRF assumption in that the size of each pixel's neighborhood is equal. The statistics between the low- and high-resolution images at each position is learnt in a nonparametric way by a number of training pairs. Similar to [3], the features on a highfrequency image are inferred from the parent structure by nearest neighbor searching. They also discuss the limits on super resolution and how to break them in their method [2]. The results in [1] appear to be noisy at places. Moreover, some global properties of a face, such as explicit contours, coherent illumination and symmetry are also missed.

It is interesting to note that all previous models use local feature inference or transfer in MRF without global correspondence being taken into account. Such global modeling is, however, essential for achieving good performance in face hallucination. Principal component analysis (PCA) has been successfully used in face recognition by eigenfaces [12] and face modeling by ASM and AAM [4]. We use PCA to model the global variance of facial appearance. Since patch-based nonparametric sampling has been applied in texture synthesis with encouraging results and high efficiency $[8,13,5]$, we build a patch-based Markov network, a nonparametric counterpart to the parametric one proposed in [6], to model the statistics between a local feature image and global face image. The theory and algorithm are explained in the next section.

\section{Theory and Algorithms}

\subsection{A Bayesian Formulation to Face Hallucination}

Let $I_{H}$ and $I_{L}$ denote the high- and low-resolution face images respectively. We simply choose the same method as that in [1] to compute $I_{L}$ from $I_{H}$. If $I_{L}$ is $s$ times smaller than $I_{H}, I_{L}$ is computed by

$$
I_{L}(m, n)=\frac{1}{s^{2}} \sum_{i=0}^{s-1} \sum_{j=0}^{s-1} I_{H}(s m+i, s n+j)
$$

where $s$ is always an integer with the default value 4 in this article. Equation (3) combines a smoothing step and a down-sampling step, more consistent with image formation as integration over the pixel [1]. To simplify the notation, if $I_{H}$ and $I_{L}$ are respectively $N-D$ and $M-D$ long vectors ( $M=N / s^{2}$ ), equation (3) can be rewritten as

$$
I_{L}=A I_{H}
$$

where $A=\left[\mathbf{a}_{1}, \mathbf{a}_{2}, \cdots, \mathbf{a}_{M}\right]^{T}$ is a $M \times N$ matrix with each row vector $\mathbf{a}_{i}^{T}$ smoothing a $s \times s$ block in $I_{H}$ to a pixel in $I_{L}$.

To compute $I_{H}$ from $I_{L}$ is straightforward in (4), but the inverse process is full of uncertainty, with uncountable $I_{H} \mathrm{~s}$ (almost) satisfying (4). Based on the maximum a posteriori (MAP) criterion, we find the optimal solution maximizing the posterior probability $p\left(I_{H} \mid I_{L}\right)$, i.e.,

$$
I_{H}^{*}=\arg \max _{I_{H}} p\left(I_{L} \mid I_{H}\right) p\left(I_{H}\right) .
$$

Under this framework, we should (a) build both the prior and likelihood models and (b) find the optimal solution.

\subsection{Global and Local Modeling of Face}

Note that in equation (5) exists the prior distribution of the face image $p\left(I_{H}\right)$. Current face prior models either capture common properties of face such as eigenfaces [12] and 
AAM [4], or represent individual characteristics such as local features [1]. But both of them are required in face hallucination. We develop a mixture model combining a global parametric model called global face image $I_{H}^{g}$ carrying common facial properties, and a local nonparametric one called local feature image $I_{H}^{l}$ recording local individualities, illustrated in Figure 2. The high-resolution face image is naturally a composition of them,

$$
I_{H}=I_{H}^{l}+I_{H}^{g}
$$

Since $I_{L}$ is the low-frequency part of $I_{H}$, the global face $I_{H}^{g}$ contributes the main part of $A I_{H}$ and the local feature $I_{H}^{l}$ lies on the high-frequency band. Mathematically,

$$
A I_{H}^{g}=A I_{H}, A I_{H}^{l}=0 .
$$

In this way, the prior model of face is decomposed to

$$
p\left(I_{H}\right)=p\left(I_{H}^{l}, I_{H}^{g}\right)=p\left(I_{H}^{l} \mid I_{H}^{g}\right) p\left(I_{H}^{g}\right) .
$$

Now we shall reformulate the MAP problem (5) under this mixture face model. The likelihood $p\left(I_{L} \mid I_{H}\right)$ is simply regarded as a soft constraint to $I_{H}$, and exhibits a Gaussian form if each pixel on $I_{L}$ is identically treated [1]

$$
p\left(I_{L} \mid I_{H}\right)=\frac{1}{Z} \exp \left\{-\left\|A I_{H}-I_{L}\right\|^{2} / \lambda\right\},
$$

where $Z$ is a normalization constant, $\lambda$ scales the variance and $\|x\|^{2}=x^{T} x$ throughout this paper. Based on (7), (9) can be rewritten as

$$
p\left(I_{L} \mid I_{H}\right)=\frac{1}{Z} \exp \left\{-\left\|A I_{H}^{g}-I_{L}\right\|^{2} / \lambda\right\}=p\left(I_{L} \mid I_{H}^{g}\right) .
$$

From equation (8) and (10), the MAP inference problem (5) can be transferred to

$$
I_{H}^{*}=\arg \max _{I_{H}^{g}, I_{H}^{l}} p\left(I_{L} \mid I_{H}^{g}\right) p\left(I_{H}^{g}\right) p\left(I_{H}^{l} \mid I_{H}^{g}\right) .
$$

Obviously $p\left(I_{L} \mid I_{H}^{g}\right) p\left(I_{H}^{g}\right)$ and $p\left(I_{H}^{l} \mid I_{H}^{g}\right)$ sequentially constrain $I_{H}^{g}$ and $I_{H}^{l}$. The solution strategy is naturally divided into two steps. In the first step we leave $I_{H}^{l}$ apart and obtain the optimal global face $I_{H}^{g *}$ by maximizing $p\left(I_{L} \mid I_{H}^{g}\right) p\left(I_{H}^{g}\right)$. In the second stage the optimal local feature image $I_{H}^{l *}$ is computed by maximizing $p\left(I_{H}^{l} \mid I_{H}^{g}\right)$. Finally $I_{H}^{*}=I_{H}^{g *}+I_{H}^{l *}$ is our desired result.

\subsection{Global Modeling: A Linear Parametric Model}

We apply PCA to modeling the global face image $I_{H}^{g}$. Given a set of training face images $\left\{I_{H}^{(i)}\right\}_{i=1}^{k}$, we can compute the eigenvectors $\left\{\mathbf{b}_{i}\right\}_{i=1}^{l}\left(\mathbf{b}_{\mathbf{i}} \in \mathbf{R}^{N}\right)$, eigenvalues $\left\{\sigma_{i}^{2}\right\}_{i=1}^{l}$ and mean face $\mu$ with $l$ the reduced dimension. The orthogonal eigenvectors construct the eigen-subspace $\Omega=\operatorname{span}\left(\mathbf{b}_{1}, \cdots, \mathbf{b}_{l}\right) \sim \mathbf{R}^{l}$. Thus $I_{H}^{g}$ is in fact the reconstructed image of $I_{H}$ in $\Omega$

$$
I_{H}^{g}=B X+\mu, X=B^{T}\left(I_{H}-\mu\right),
$$

where $B=\left[\mathbf{b}_{1}, \cdots, \mathbf{b}_{l}\right]_{N \times l}$, and $X=\left(x_{1}, \cdots, x_{l}\right)^{T}$ is a vector in $\Omega$. Intuitively $I_{H}^{g}$ is linearly controlled by $x_{i}$ s with corresponding eigenvectors $\mathbf{b}_{i}$ s. Since the eigenvectors are analyzed from the training data representing the irrelevant common face properties such as lighting, scale and pose, $I_{H}^{g}$ retains the common features of $I_{H}$ with individuality lost.

The distribution of $I_{H}^{g}$ can be purely replaced by $X$ based on (12). Maximizing $p\left(I_{L} \mid I_{H}^{g}\right) p\left(I_{H}^{g}\right)$ in (11) is equivalent to maximizing $p\left(I_{L} \mid X\right) p(X)$. The prior $p(X)$ is simply assumed as Gaussian:

$$
p(X)=\frac{1}{Z^{\prime}} \exp \left\{-X^{T} \Lambda^{-1} X\right\},
$$

where $\Lambda=\operatorname{diag}\left(\sigma_{1}^{2}, \cdots, \sigma_{l}^{2}\right)$ and $Z^{\prime}$ is a normalization constant. The likelihood (10) is replaced by

$$
p\left(I_{L} \mid X\right)=\frac{1}{Z} \exp \left\{-\left\|A(B X+\mu)-I_{L}\right\|^{2} / \lambda\right\} .
$$

To maximize $p\left(I_{L} \mid X\right) p(X)$ is

$$
X^{*}=\arg \min _{X} \lambda X^{T} \Lambda^{-1} X+\left\|A(B X+\mu)-I_{L}\right\|^{2},
$$

with solution:

$$
X^{*}=\left(B^{T} A^{T} A B+\lambda \Lambda^{-1}\right)^{-1} B^{T} A^{T}\left(I_{L}-A \mu\right) .
$$

The optimal global face image $I_{H}^{g *}=B X^{*}+\mu$. Since matrix $B, \Lambda$ and $\mu$ are learnt by PCA, and $A$ is constant as a smoothing and down-sampling function, all matrices on the right side of (16) can be off-line computed and $I_{H}^{g *}$ is calculated very fast input $I_{L} . I_{H}^{g *}$ is very close to human face with some smoothness, which will be improved by the local model in next subsection.

\subsection{Local Modeling: Patch-based Nonparametric Markov Network}

In most cases PCA is used, the random variable is regarded as a composition of two parts: principal components and independent residual. But in our mixture modeling, the residual $I_{H}^{l}=I_{H}-I_{H}^{g}$ is the highest frequency component, relying on rather than independent to the lower frequency part $I_{H}^{g}$ [6]. To carefully model $p\left(I_{H}^{l} \mid I_{H}^{g}\right)$, we combine Markov network [6] and "Image Analogies" [7] to generate a new patch-based nonparametric Markov network, in which the statistics between two images are modelled by connected patches in a nonparametric way. The function of the Markov network is shown in Figure 2.

Let $I_{H}^{l}$ and $I_{H}^{h}$ be decomposed to square patches with size $w+h . I_{H}^{l}(m, n)$ and $I_{H}^{g}(m, n)$ denote patches centered at position $\left(\left(m+\frac{1}{2}\right) w,\left(n+\frac{1}{2}\right) w\right)$ respectively. For simplification, we replace $(m, n)$ by a vector $\vec{v}$. For patch $I_{H}^{l}(\vec{v})$, its neighboring patches are $N_{H}^{l}(\vec{v})=\left\{I_{H}^{l}\left(\vec{v}+\Delta_{x}\right), I_{H}^{l}(\vec{v}-\right.$ $\left.\left.\Delta_{x}\right), I_{H}^{l}\left(\vec{v}+\Delta_{y}\right), I_{H}^{l}\left(\vec{v}-\Delta_{y}\right)\right\}$ with overlapping size $h$ and the overlapping area $S(\vec{v})=I_{H}^{l}(\vec{v}) \cap N_{H}^{l}(\vec{v})$, where $\Delta_{x}=$ $(1,0), \Delta_{y}=(0,1)$. Such circumstance varies slightly at the image boundary. $I_{H}^{l}$ can be reconstructed by superposing 


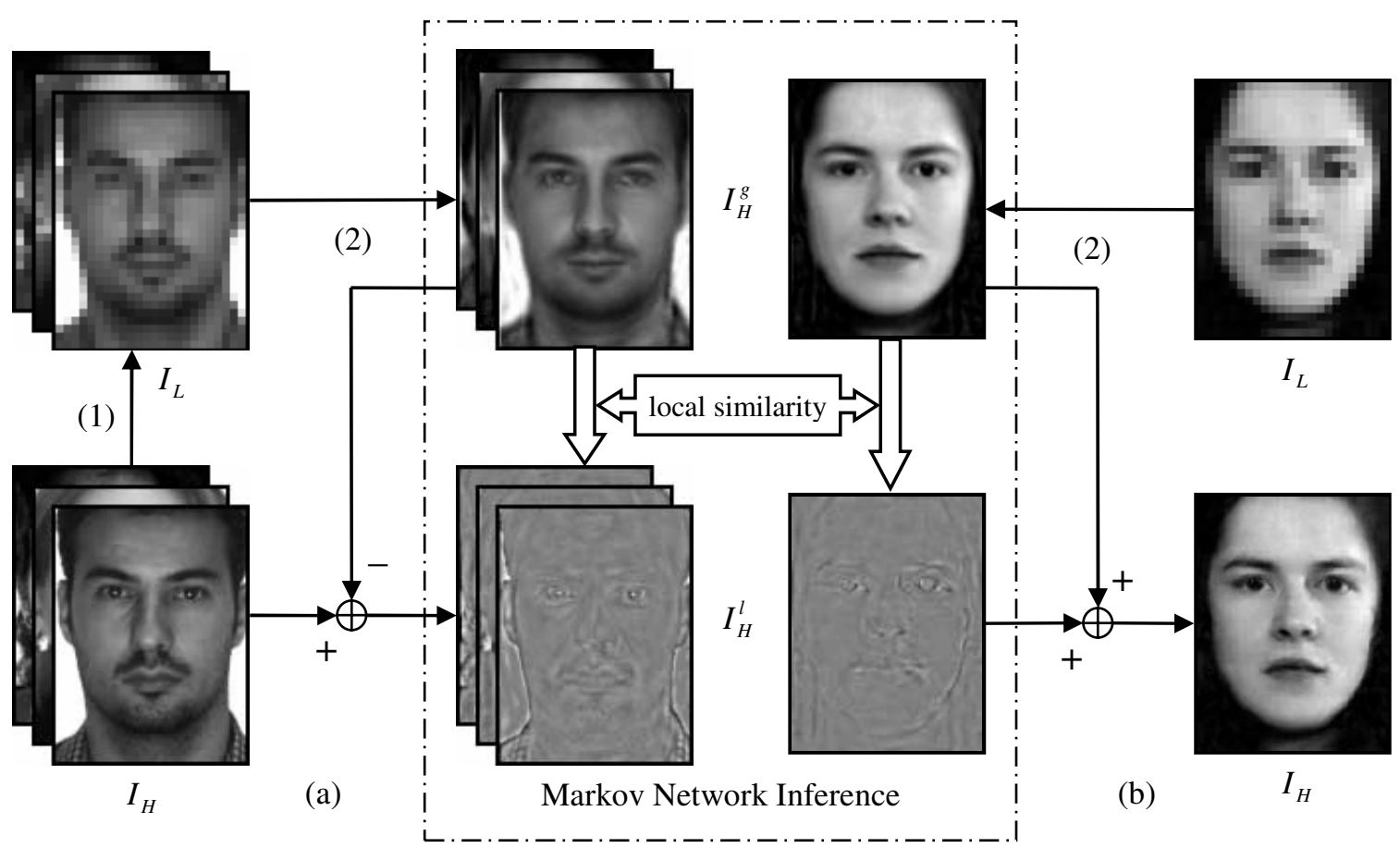

Figure 2. The function of Markov network in our model. (a) is the training process and (b) the hallucinating process. (1): smooth and down-sampling. (2): MAP inference to get the optimal global face $I_{H}^{g *}$. The Markov network finds the optimal local feature image $I_{H}^{l *}$ by energy minimization.

its patches, with pixels in the overlapping area blended. The patches at the same position, $I_{H}^{l}(\vec{v})$ and $I_{H}^{g}(\vec{v})$, are connected between $I_{H}^{l}$ and $I_{H}^{g}$. Thus all patches in these two images constitute a network, as illustrated in Figure 3.

Suppose $I_{H}^{l-}(\vec{v})$ denotes all patches on $I_{H}^{l}$ except patch $I_{H}^{l}(\vec{v})$. We assume that the above network is a Markov network by defining:

$$
p\left(I_{H}^{l}(\vec{v}) \mid I_{H}^{l-}(\vec{v}), I_{H}^{g}\right)=p\left(I_{H}^{l}(\vec{v}) \mid N_{H}^{l}(\vec{v}), I_{H}^{g}(\vec{v})\right),
$$

This definition is equivalent to that in [6], but not homogeneous because the conditional density function of each patch is not identical. Suppose $p\left(I_{H}^{l}(\vec{v}) \mid N_{H}^{l}(\vec{v}), I_{H}^{g}(\vec{v})\right)$ a Gibbs distribution

$p\left(I_{H}^{l}(\vec{v}) \mid N_{H}^{l}(\vec{v}), I_{H}^{g}(\vec{v})\right) \propto \exp \left\{-E_{G}\left(I_{H}^{l}(\vec{v}), N_{H}^{l}(\vec{v}), I_{H}^{g}(\vec{v})\right)\right\}$

where $E_{G}(\cdot)$ is the Gibbs potential function to describe how likely a patch $I_{H}^{l}(\vec{v})$ connects to $I_{H}^{g}(\vec{v})$ and is surrounded by $N_{H}^{l}(\vec{v})$. It is natural to decouple it into two terms concerning $N_{H}^{l}(\vec{v})$ and $I_{H}^{g}(\vec{v})$ independently,

$$
\begin{aligned}
& E_{G}\left(I_{H}^{l}(\vec{v}), N_{H}^{l}(\vec{v}), I_{H}^{g}(\vec{v})\right) \\
= & E_{G}^{i n t}\left(I_{H}^{l}(\vec{v}), N_{H}^{l}(\vec{v})\right)+E_{G}^{e x t}\left(I_{H}^{l}(\vec{v}), I_{H}^{g}(\vec{v})\right) \\
\equiv & E_{G}^{i n t}(\vec{v})+E_{G}^{e x t}(\vec{v})
\end{aligned}
$$

where $E_{G}^{i n t}(\vec{v})$ is the internal potential function that describes the neighboring statistics between patches inside $I_{H}^{l}$, and $E_{G}^{e x t}(\vec{v})$ is the external potential function that represents the connecting statistics between connecting patches in $I_{H}^{l}$ and $I_{H}^{g}$.

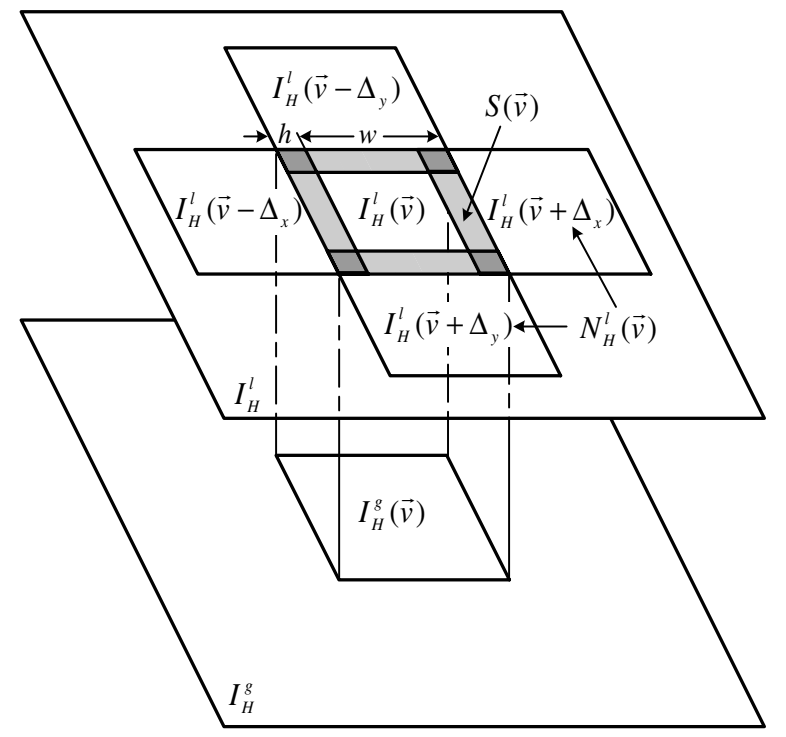

Figure 3. Illustration of the patch-based Markov network.

The external potential function $E_{G}^{e x t}(\vec{v})$ is modeled upon training examples. Suppose at position $\vec{v}$ we have $k$ training pairs $\left\{I_{H}^{l(i)}(\vec{v}), I_{H}^{g(i)}(\vec{v})\right\}_{i=1}^{k}$, then

$$
E_{G}^{e x t}(\vec{v})=\frac{1}{\lambda^{\prime}} \sum_{i=1}^{k} \delta\left[I_{H}^{l}(\vec{v})-I_{H}^{l(i)}(\vec{v})\right] d^{2}\left[I_{H}^{g}(\vec{v}), I_{H}^{g(i)}(\vec{v})\right],
$$

where $\delta(\cdot)$ is the dirac function, $d(\cdot)$ is the distance metric between two patches in $I_{H}^{g}$, and $\lambda^{\prime}$ scales the variance. The distance plays a crucial role in nonparametric models, and can be chosen as SSD (sum of squared differences) on 
pixel value or on feature images such as pyramid and parent structures $[3,1,7,8]$. Since the signals in the highfrequency band depend on those in the lower frequency band [6], here we define the distance on the Laplacian image $L_{H}^{g}$ of $I_{H}^{g}$, which in fact represents the middle-frequency band of the face image. The squared distance between $I_{H}^{g}(\vec{v})$ and $I_{H}^{g(i)}(\vec{v})$ is

$$
d^{2}\left[I_{H}^{g}(\vec{v}), I_{H}^{g(i)}(\vec{v})\right]=\left\|L_{H}^{g}(\vec{v})-L_{H}^{g(i)}(\vec{v})\right\|^{2} .
$$

Equation (20) and (21) form a nonparametric distribution: compare the given patch $I_{H}^{g}(\vec{v})$ with training patches $\left\{I_{H}^{g(i)}(\vec{v})\right\}_{i=1}^{k}$, then the patch $I_{H}^{l(i)}(\vec{v})$ with $I_{H}^{g(i)}(\vec{v})$ close to $I_{H}^{g}(\vec{v})$ is most probable to be chosen as $I_{H}^{l}(\vec{v})$. This is the key principle of modeling example-based conditional density [3].

The internal potential function $E_{G}^{i n t}(\vec{v})$ is introduced to make neighboring patches well connected. Since the neighboring patches overlap each other, it enforces the common part of the abutting patches to be as similar as possible in the overlapping area. Mathematically it is defined by:

$$
E_{G}^{i n t}(\vec{v})=\frac{1}{\lambda^{\prime \prime}} \sum_{\mathbf{u} \in S(\vec{v})}\left[I_{H}^{l}(\mathbf{u})-N_{H}^{l}(\mathbf{u})\right]^{2},
$$

where $I_{H}^{l}(\mathbf{u})$ is the pixel value of $I_{H}^{l}$ and $\lambda^{\prime \prime}$ scales the variance. The total energy $E_{M N}$ of the Markov network is the sum of each patch's energy:

$$
E_{M N}=\sum_{\vec{v}}\left(E_{G}^{i n t}(\vec{v})+E_{G}^{e x t}(\vec{v})\right) .
$$

We have mentioned in 3.2 that after the optimal global face $I_{H}^{g *}$ is obtained, the optimal local feature $I_{H}^{l *}$ is found to maximize $p\left(I_{H}^{l} \mid I_{H}^{g *}\right)$. This is equivalent to minimizing the total energy $E_{M N}$ of the Markov network, i.e.

$$
I_{H}^{l *}=\arg \min _{I_{H}^{l}} E_{M N}
$$

Finding the global minimum of $E_{M N}$ is not trivial. If there are totally $R \times C$ patches in $I_{H}^{l}$ and $k$ pairs of training images, the solution space is as huge as $k^{R \times C}$ ! A greedy algorithm which sequentially finds $I_{H}^{l *}(\vec{v})$ by minimizing $E_{G}(\vec{v})$ such as [7], can only find a local optimum. We use simulated annealing which has been successfully used in many areas to find a global or satisfactory optimum. In every step when we need to flip $I_{H}^{l}(\vec{v})$, each candidate patch in $\left\{I_{H}^{l(i)}(\vec{v})\right\}_{i=1}^{k}$ is selected as $I_{H}^{l}(\vec{v})$ with probability proportional to

$$
\exp \left\{-\left(E_{G}^{i n t}(\vec{v})+E_{G}^{e x t}(\vec{v})\right) / T\right\},
$$

where $T$ is the temperature. In each loop, every patch in image $I_{H}^{l}$ is sequentially randomly flipped. By gradually decreasing $T$ to zero, $I_{H}^{l}$ will converge to the global optimal $I_{H}^{l *}$. The pseudo code is listed below.

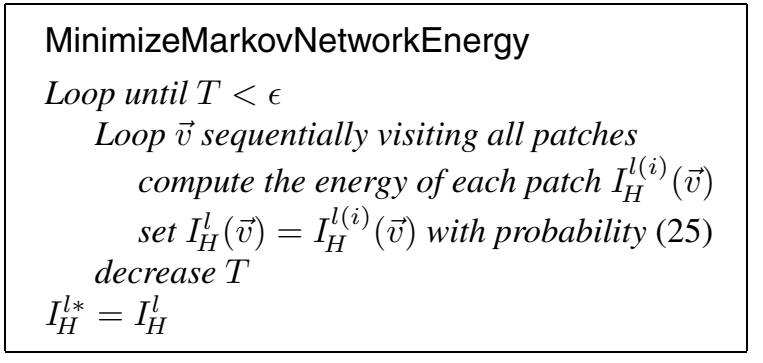

\section{Experimental Results}

Our experiments are conducted with a large number of frontal face images in the FERET data set [10], AR data set [9] and other collections, involving all kinds of races, illuminations and types of face. We select 1114 images as the training data, and other 39 images as the test data. Before experiment we manually align the face image with five points: the centers of the eyeballs, the tip of the nose and the corners of the mouth. The centers of the eyeballs are used to calibrate the orientation, and the five together are used to calibrate the scale. We do not choose the affine warp to retain the shape of a face. After a similarity transform, each image is aligned to a canonical $96 \times 128$ pixel image. The high-resolution image is smoothed and down-sampled to a low-resolution $24 \times 32$ image by (3).

We use standard SVD [11] to do principal component analysis of the training images. The dimension of the face images is reduced to 200 to retain $97 \%$ of the eigenvalue total. Since the number of the training data is much larger than the reduced dimension, the inverse of the covariancelike matrix in equation (16) does exist. In solution (16), $\lambda$ controls the balance between the similarity of $I_{H}^{g}$ to input $I_{L}$ and that to mean face $\mu$. We find values in $0.05 \sim 0.2$ appropriate for $\lambda$ and we choose 0.1 in the experiment. Some obtained $I_{H}^{g *}$ s are displayed in Figure 4(b). Compared with the low-resolution input, such solutions have more explicit contours and edges, because the principal components record the common variance of face image.

Further we apply the patch-based nonparametric Markov network to infer the optimal local feature image. We choose $w=5, h=2$ as the patch size and overlapping size. In fact, the results vary little if $w$ is chosen between 4 and 6 . The parameters $\lambda^{\prime}$ and $\lambda^{\prime \prime}$ control the tradeoff between the external and internal potentials. We simply choose $\lambda^{\prime}=\lambda^{\prime \prime}=3 \mathrm{wh}$. The initial value of the temperature $T$ is set to 1 . In the simulated annealing process, $T$ gradually decreases $10 \%$ at each time step. Finally we get the hallucinated face $I_{H}^{*}$ by summing $I_{H}^{l *}$ and $I_{H}^{g *}$ up, shown in Figure 4(c). We may see that these results suit the three goals of face hallucination very well, visually very close to the original high images, Figure 4(d). Note that each part of the hallucinated face such as the eyes and nose, are different from that in the original image but as detailed as that in the real face image. The runtime of computing the global face is less than 1 second 

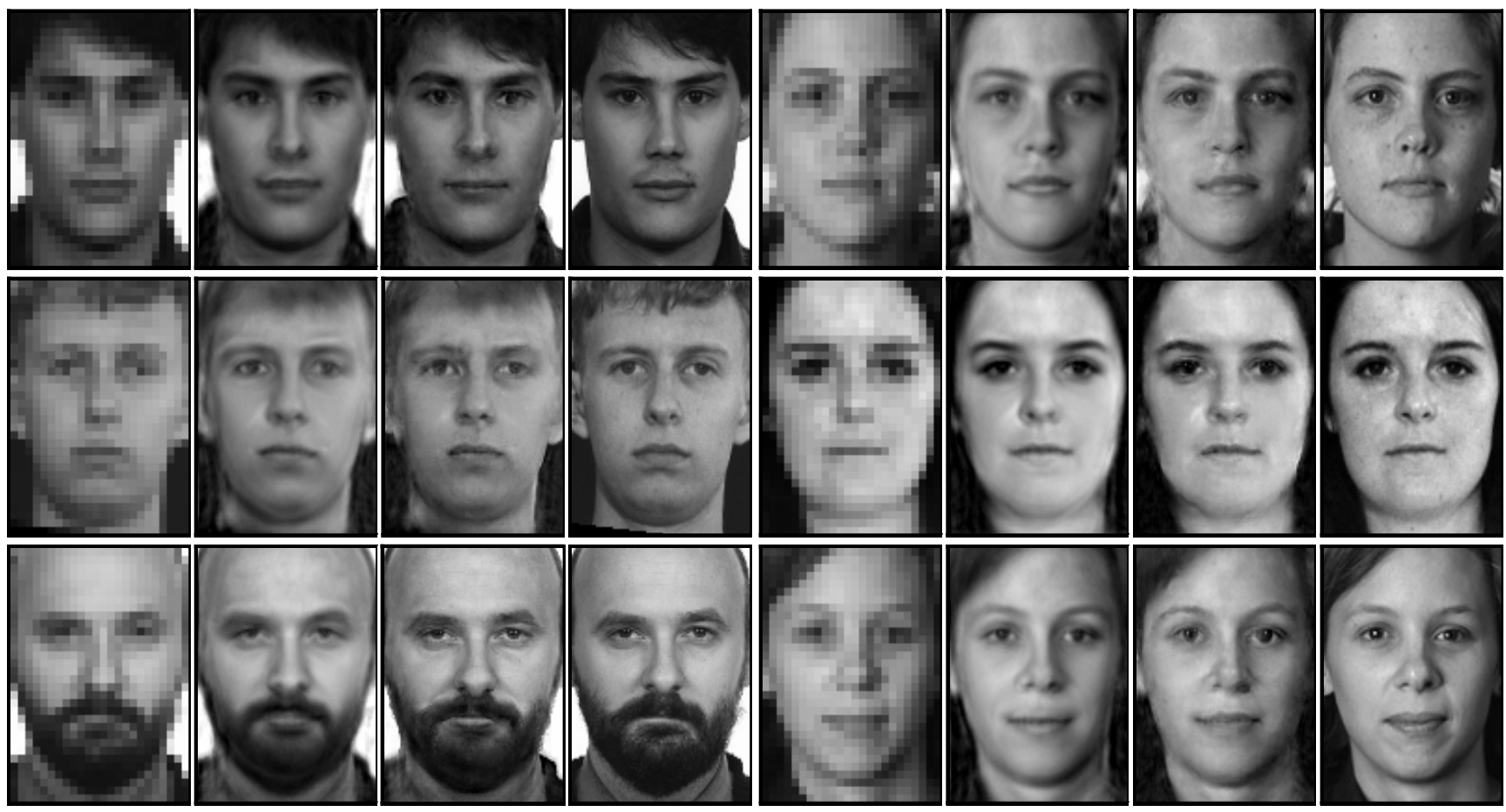

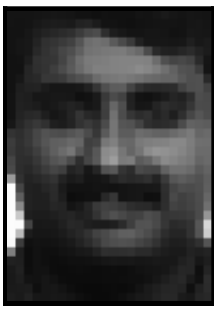

(a)
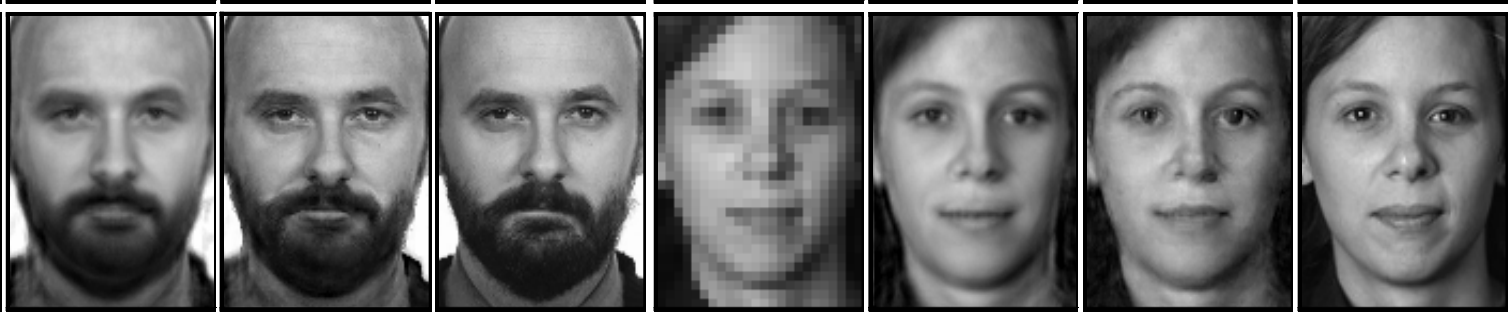

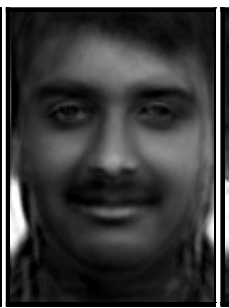

(b)

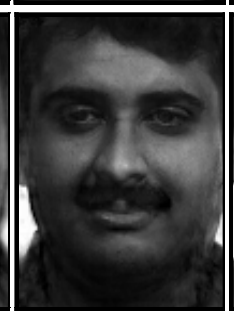

(c)

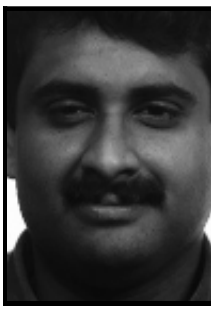

(d)

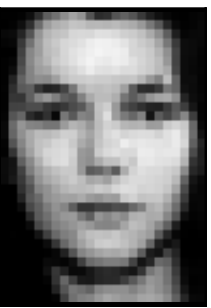

(a)

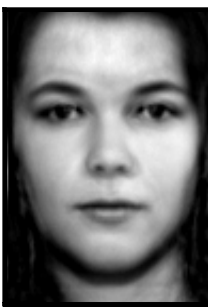

(b)

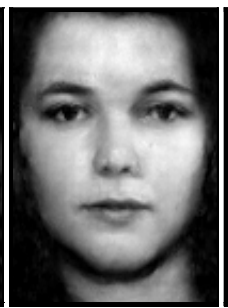

(c)

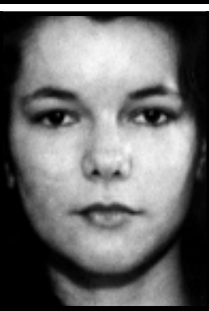

(d)

Figure 4. The hallucination results. (a) is the low-resolution $24 \times 32$ input. (b) is the inferred global face $I_{H}^{g *}$ from (a). (c) is the final result $I_{H}^{*}=I_{H}^{g *}+I_{H}^{l *} . I_{H}^{l *}$ is inferred from $I_{H}^{g *}$ by Markov network. (d) is the original high-resolution $96 \times 128$ image.

and the simulated annealing takes about half a minute.

We compare our algorithm with existing methods in Figure 5 for another group of individuals, using the same training data set. Those methods include (c) Cubic B-Spline , (d) Hertzmann's "Image Analogy" (We implement it in a patch-based way, also a nonparametric counterpart of Freeman's Markov network.) and (e) Baker's method. (d) is locally close to the face image but lacks global face features such as symmetry. (e) has too much noise also with global features missed. It is obvious that the result of our model (b) with both the global and local face information taken into account, is closer to the original face (f) with very high image qualities.

\section{Discussion and Conclusion}

Under MAP criterion, the kernel of face hallucination problem is how to model the low-resolution constraint and the appearance of face. A parametric model is proper to capture the common structural properties whereas a nonparametric model is appropriate to represent local and in- dividual patterns. It is natural for us to combine them in face hallucination to integrate their merits. A linear model is used to model the global variance of face and the lowresolution constraint to achieve robustness and efficiency. We do not choose more complex models as the global face model because the modeling error can be compensated in the local model. We devise a patch-based nonparametric Markov network, a combination of $[6,7]$, to learn the relationship between local facial feature and global face. Nonparametric ensures it accuracy and patch-based endows it with high efficiency. The simulated annealing method here is very similar to the Gibbs sampling in [14], but converges more quickly. This method can also be expanded to general super-resolution problem.

To hallucinate high-quality face images, we develop a two-step statistical inference model which integrates both a global parametric linear model and a local nonparametric one. The effectiveness of our approach is demonstrated by extensive experiments. 

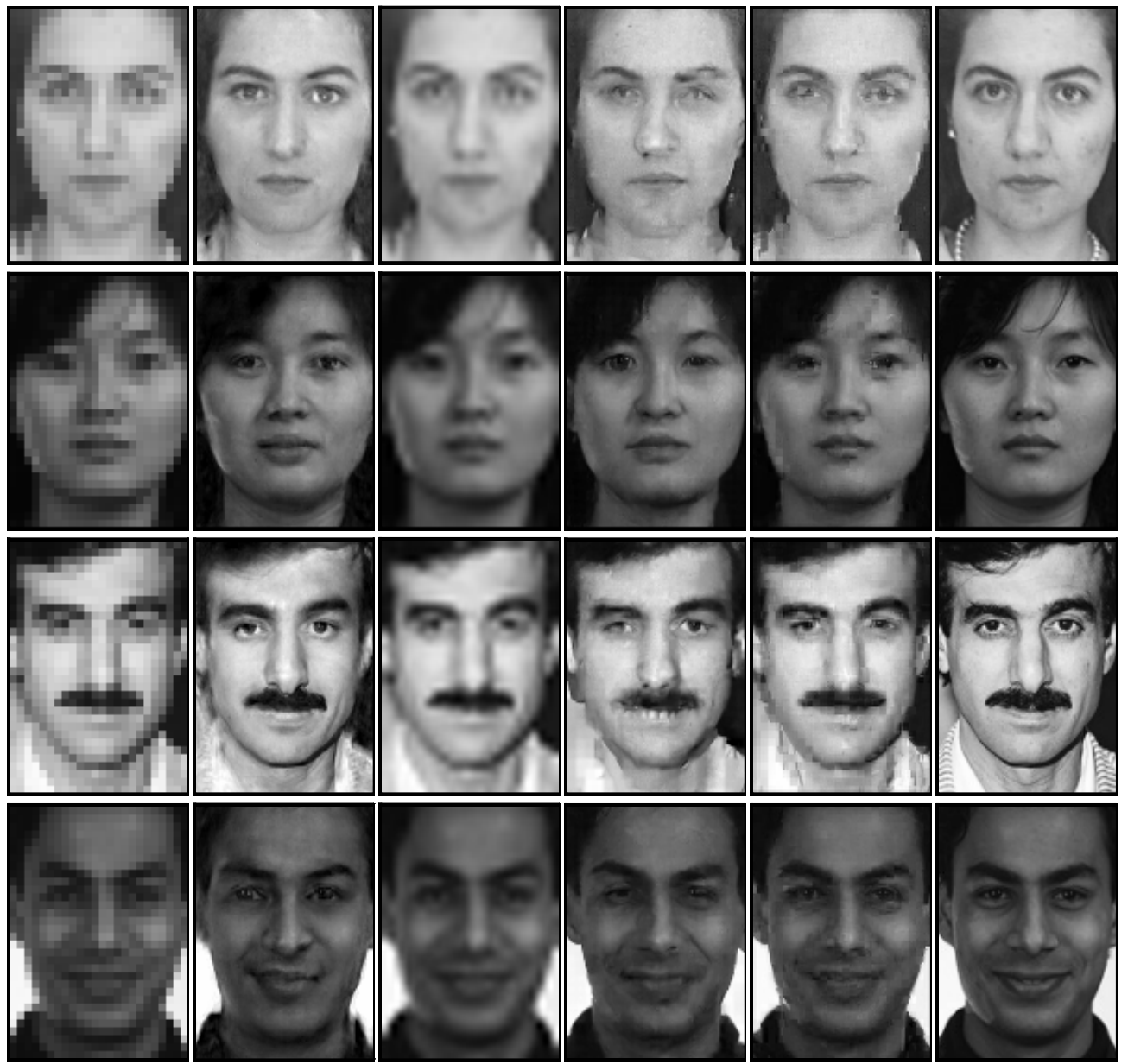

(a) Input $24 \times 32$
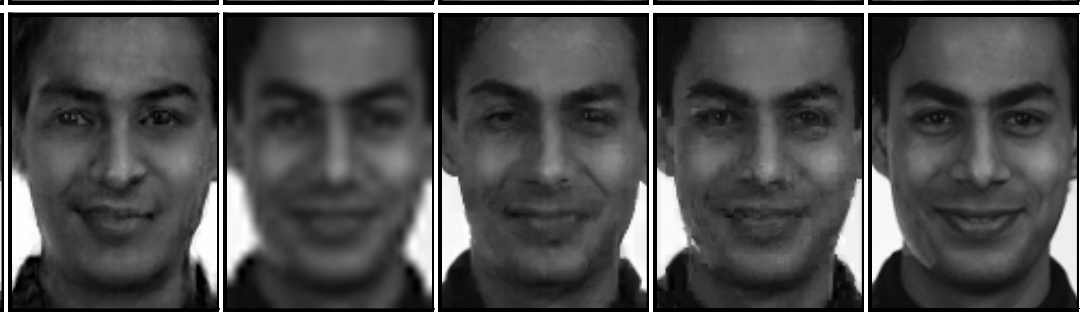

(b) Our method

(c) Cubic B-Spline (d) Hertzmann et al.

(e) Baker et al. (f) Original $96 \times 128$

Figure 5. Comparison between our method and others.

\section{References}

[1] S. Baker and T. Kanade. Hallucinating faces. Fourth International Conference on Automatic Face and Gesture Recognition, March 2000.

[2] S. Baker and T. Kanade. Limits on super-resolution and how to break them. IEEE conference on Computer Vision and Pattern Recognition, June 2000.

[3] J. D. Bonet. Multiresolution sampling procedure for analysis and synthesis of texture images. Proceedings of SIGGRAPH 97, pages 361-368, August 1997.

[4] T. Cootes and C. Taylor. Statistical models of appearance for computer vision. Technical report, University of Manchester, 2000.

[5] A. Efros and W. Freeman. Quilting for texture synthesis and transfer. Proceedings of SIGGRAPH 2001, August 2001.

[6] W. Freeman and E. Pasztor. Learning low-level vision. 7-th International Conference on Computer Vision, pages 11821189, 1999.

[7] A. Hertzmann, C. Jacobs, N. Oliver, B. Curless, and D. Salesin. Image analogies. Proceedings of SIGGRAPH 2001, August 2001.
[8] L. Liang, C. Liu, Y. Xu, B. Guo, and H. Shum. Real-time texture synthesis by patch-based sampling. ACM Transaction on Graphics, 2001.

[9] A. Martinez and R. Benavente. The ar face database. Technical report, CVC Techinical Report No.24, June 1998.

[10] P. Philips, H. Moon, P. Pauss, and S. Rivzvi. The feret evaluation methodology for face-recognition algorithms. In Proceedings of CVPR'97, pages 137-143, 1997.

[11] W. Press, S. Teukolsky, W. Vetterling, and B. Flannery. Numerical Recipes in $C$. Cambridge University Press, second edition, 1992.

[12] M. Turk and A. Pentland. Eigenfaces for recognition. Journal of Cognitive Neurosciences, 3:71-86, 1991.

[13] Y. Xu, B. Guo, and H. Shum. Chaos mosaic: fast and memory efficient texture synthesis. Technical report, In Microsoft Research Technical Report MSR-TR-2000-32, April 2000.

[14] S. Zhu, Y. Wu, and D. Mumford. Minimax entropy principle and its application to texture modeling. Neural Computation, 9(8), November 1997. 